\title{
Théologiques
}

\section{Bandelettes pour Jacques Derrida}

\section{Jacques Julien}

Volume 12, numéro 1-2, 2004

Le Soi dans tous ses états

URI : https://id.erudit.org/iderudit/011565ar

DOI : https://doi.org/10.7202/011565ar

Aller au sommaire du numéro

Éditeur(s)

Faculté de théologie et de sciences des religions, Université de Montréal

ISSN

1188-7109 (imprimé)

1492-1413 (numérique)

Découvrir la revue

Citer cet article

Julien, J. (2004). Bandelettes pour Jacques Derrida. Théologiques, 12(1-2),

245-268. https://doi.org/10.7202/011565ar

Tous droits réservés $\subset$ Faculté de théologie et de sciences des religions, Université de Montréal, 2005
Ce document est protégé par la loi sur le droit d'auteur. L'utilisation des services d'Érudit (y compris la reproduction) est assujettie à sa politique d'utilisation que vous pouvez consulter en ligne.

https://apropos.erudit.org/fr/usagers/politique-dutilisation/
Cet article est diffusé et préservé par Érudit.

Érudit est un consortium interuniversitaire sans but lucratif composé de l’Université de Montréal, l'Université Laval et l'Université du Québec à Montréal. Il a pour mission la promotion et la valorisation de la recherche. https://www.erudit.org/fr/ 


\title{
Bandelettes \\ pour Jacques Derrida
}

Jacques Julien, post-doctorant Faculté de théologie et de sciences des religions Université de Montréal

\begin{abstract}
En grec c'est aussi un mot superbe, phulakè: la garde mais aussi le garde, la sentinelle [...]. Et "phylactère» vient de là. Tu sais ce que ça représente pour nous, enfin pour nous les Juifs. Mais tu comprendras pourquoi tout à l'heure j'ai bondi à lire cette définition dans mon dictionnaire: "phylaktèrion... lieu pour garder, poste, corps de garde... préservatif... talisman, amulette... chez les Juifs, pancarte qu'on portait suspendue au cou et où étaient inscrits des versets de la loi mosaïque...» ( $\mathrm{La}$ carte postale $1980,9^{1}$ )
\end{abstract}

\section{Le corps de Jacques Derrida}

Des voix nombreuses, importantes et plurielles se sont élevées à la mémoire de Jacques Derrida. Le volumineux cahier de L'Herne (Mallet et Michaud 2004) qui lui a été consacré est venu s'ajouter aux quatre forts volumes des décades de Cerisy tenues autour de son œuvre: Les fins de l'homme (Collectif 1981), Le passage des frontières (Collectif 1994), L'animal autobiographique (Collectif 1999) et La démocratie à venir (Collectif 2004) - tandis que la revue Europe lui avait consacré son numéro de mai 2004. Devant cette littérature imposante, comment aborder, brièvement, dans le cadre de cet article, le corps de Jacques Derrida devenu maintenant le corpus derridien? Par où le toucher, lui qui offre si peu de prise, même à l'affection? Geoffrey Bennington l'a noté:

Ce travail remarquable par sa diversité et sa consistance, ses puissances de dispersion et de rassemblement, sa formation (PS [Psyché], 9), ne supporte pas de division en styles ou en périodes: même l'idée assez répandue selon

1. Les œuvres de Derrida sont citées par leur titre et leur année de parution, sans mention de l'auteur. 
laquelle il y aurait d'abord des textes très philosophiques et, après Glas (1974), une tendance plus "littéraire " et moins "sérieuse » est douteuse quant à sa vérité empirique et sans pertinence pour notre compréhension (Bennington et Derrida 1991, 16-17).

Il semble que nous soyons renvoyés et maintenus à de certaines marges, à un certain abord sans abordage, à l'entourage ou aux alentours de l'œuvre de Jacques Derrida. Cependant, lui-même a indiqué une voie, une certaine ouverture: "En fait, vous savez, il faut surtout lire et relire ceux dans les marges desquels j'écris, les "livres" dans les marges et entre les lignes desquels je dessine et déchiffre un texte qui est à la fois très ressemblant et tout autre, que j'hésiterais même, pour des raisons évidentes, à appeler fragmentaire...» ( «Implications", dans Positions 1972, 12). Le numéro spécial d'Études françaises, Derrida lecteur (Michaud et Leroux 2002) l'avait suggéré: entrer en Derrida, c'est s'engager à parcourir un champ de lectures qui est également un labyrinthe, à s'asseoir avec une longue patience dans les salles de multiples bibliothèques. Et à lire, aussi, d'une certaine façon, avec un certain tour de l'œil et de la main, parfois une marque de l'ongle sur la page, faire de la lecture une fréquentation qui soit aussi une «rumination». En langage technique moderne: pratiquer l'hypertextualité, certes, mais également cueillir, au verger de l'interprétation, les fruits de la très ancienne matrice de lecture juive (matres lectionis), talmudique ou cabalistique.

Dans sa brève allocution au moment de la mort de Hans-Georg Gadamer, Derrida se disait «tout à fait d'accord» avec le philosophe allemand qu'il citait longuement:

"Toute lecture qui cherche à comprendre n'est qu'un pas sur un chemin qui ne trouve jamais de terme. Quiconque s'engage sur ce chemin sait qu'il ne viendra jamais "à bout" de son texte; il en reçoit le coup. Quand un texte poétique l'a touché à ce point qu'il finit par “entrer” en lui et s'y reconnaître, cela ne suppose ni l'accord ni la confirmation de soi. On s'abandonne, pour se trouver. Je ne me crois pas si éloigné de Derrida, quand je souligne qu'on ne sait jamais d'avance ce que l'on sera quand on se trouvera.» [Gadamer 1991, 238] ("Wie recht er hatte! Mein Cicerone Hans-Georg Gadamer² », dans le Frankfurter Allgemeine Zeitung, 23 mars 2002)

2. «Comme il avait raison! Mon Cicérone Hans-Georg Gadamer », traduction de Jean Grondin que je remercie de m'avoir signalé ce texte. 
Posé ce contexte, je lirai donc Derrida lecteur des autres, Derrida en discussion, en échange, commerce ou dialogue. Ce dernier mot à saisir avec les pincettes des guillemets. À l'ouverture de Béliers; le dialogue ininterrompu: entre deux infinis, le poème (2003, 13), Derrida nous en prévient: "En parlant de dialogue, je me sers ici d'un mot dont j'avoue qu'il restera longtemps, pour mille raisons, bonnes ou mauvaises, [...] étranger à mon lexique, comme une langue étrangère dont l'usage appellerait des traductions inquiètes et précautionneuses.» Entre lui et Gadamer, par exemple, ç'avait été en effet un "dialogue» cahoteux, heurté, interrompu même, avec un certain fracas public et avec un certain «effet» théâtral de la philosophie. Gadamer avait compris la valeur dialogale de la halte, de l'attente même dans l'indécision. Et pour cela, écrit Derrida:

Plus que l'indécision elle-même, j'admire le respect marqué par Gadamer à l'endroit d'une indécision. Celle-ci semble interrompre ou suspendre le déchiffrement de la lecture mais en vérité elle en assure l'avenir. L'indécision tient à jamais l'attention en haleine, c'est-à-dire en vie, éveillée, vigilante, prête à s'engager dans tout autre chemin, à laisser venir, tendant l'oreille, l'écoutant fidèlement, l'autre parole, suspendue au souffle de l'autre parole et de la parole de l'autre - là même où elle pourrait sembler encore inintelligible, inaudible, intraduisible. L'interruption est indécise, elle indécide. Elle donne souffle à la question qui, loin de paralyser, met en mouvement. L'interruption libère même un mouvement infini. Dans Vérité et Méthode, Gadamer a besoin de souligner ce qu'il appelle le "caractère sans fin du dialogue ». (Béliers 2003, 37-38)

Derrida rappelle que Gadamer lui-même a nommé «à deux reprises au moins» le processus infini qui préside au dialogue et qui en décide l'issue.

Si «aucun dialogue n'a jamais vraiment été terminé ", c'est qu'un accord «véritable ", un «accord parfait entre deux personnes va à l'encontre de l'essence même de l'individualité », situation dans laquelle Gadamer reconnaît le signe de la finitude même. Je dirais que la finitude interruptrice est cela même qui appelle le processus infini. D'autre part, le "processus infini » est encore nommé deux pages plus loin pour caractériser cette fois le dialogue interminable du traducteur avec lui-même. (Béliers, 38-39)

En proie à l'indécision, à l'atermoiement, au soupçon, à la pression publique et au chantage de la réputation à maintenir, le dialogue persiste 
grâce à la promesse, cette force stabilisatrice, "ancre bien fermement fixée, qui pénètre au-delà du voile» (Hébreux 6,19):

Et la promesse: à un certain moment, grâce à l'immortalité de Gadamer, le jour certainement promis viendrait (car la promesse est un événement en soi, même si elle n'est pas tenue) où notre Auseinandersetzung [explication] aurait enfin lieu. Grâce à cette certitude et cette promesse, toutes nos rencontres, en tout cas d'après ma perception, étaient marquées d'une souriante amitié, de bonheur et de confiance. Peut-être cette situation heureuse faisait-elle partie - je voudrais tellement y croire - du « secret de la rencontre ", dont parle Celan dans le "Méridien », le poète que nous admirions tous deux, même si nous le lisions différemment. ("Wie recht er hatte! Mein Cicerone Hans-Georg Gadamer» 2002)

\section{Inscrire la figure}

Vous lisez donc ici un discours de circonstance. Tel que Derrida lui-même en a prononcé, écrit, publié plusieurs: Béliers (2003, pour Gadamer), Schibboleth pour Paul Celan (1986) et puis le discours de réception du prix Adorno, à Francfort, le 22 septembre 2001 (Fichus 2002). Écho d'un autre discours de réception (Le Méridien) prononcé par Paul Celan (voir 1971) à l'occasion d'un autre prix (le prix Georg-Büchner) qui lui avait été remis. Ce texte du poète étant la fin d'une explication qui n'eut jamais lieu entre Heidegger et lui, poète juif, autour du nom d'un autre lieu, Auschwitz et de ce dont ce nom tient lieu. Explication, discussion, rencontre de l'Allemagne, de la langue allemande et de son "esprit» (De l'esprit: Heidegger et la question 1987). Le Méridien, "[...] "discours" comme on dit: une allocution de circonstance, et datée" (Schibboleth 1986, 16). Prenant la parole à l'évocation de ces contemporains aimés, Jacques Derrida s'est fortement ancré, comme eux, dans la circonstance, dans le lieu, le temps, l'événement, la langue et, plus précisément, le poème et la traduction.

C'est que la circonstance n'est pas accessoire et elle n'est surtout pas un accident. La marque, l'inscription de la circonstance est une date. On en trouve la formulation parfaite dans Le Méridien de Paul Celan. Une date, une annonce, celle-ci, par exemple, entre mille: "dans la nuit de vendredi 8 à samedi 9 octobre Jacques Derrida est mort à l'âge de 74 ans ». Le stylet de la mémoire doit inscrire cette date dans le cœur des héritiers. Cependant, "Comment dater ce qui ne se répète pas si la 
datation fait aussi appel à quelque forme de retour, si elle rappelle dans la lisibilité une répétition? Mais comment dater autre chose que cela même qui jamais ne se répète?" (Schibboleth 1986, 13). Par ailleurs, même si «telle date aura pu s'écrire, seule, unique, soustraite à la répétition », il n'en demeure pas moins que «cette propriété absolue peut aussi être transcrite, exportée, déportée, expropriée, réappropriée, répétée dans sa singularité absolue. Il le faut même si elle doit s'exposer, risquer de se perdre dans une lisibilité » (Schibboleth 1986, 18). Juive était la date. Située aussi dans le sexe du Juif, dans cet «une fois » de la circoncision, de la marque unique, du « je t'ai choisi, tu es mien", même à ton corps défendant.

Cette marque profonde dans le temps fait se lever la question: qu'estce qu'un événement? Et puis: «dire l'événement, est-ce possible?»(Derrida, Soussana et Nouss 2001). Plus particulièrement lorsqu'il s'agit de la mort: est-elle l'événement par essence (et ce mot essence, si éthéré, comme il détonne dans ce contexte du vivant et de l'animal) ? Dire l'événement de la mort de Jacques Derrida est-il possible? C'était la volonté dernière du philosophe que rien ne soit dit à l'occasion de ses funérailles. Qu'il ne se dise rien. Ou que rien ne se dise ou que rien se dise: on pourra faire tourner sous toutes ses faces cette étrange monnaie qui n'achète rien.

En ce qui nous concerne, filles et fils, héritiers, séminaristes de ses innombrables séminaires partout dans le monde, comment inscrire la marque dans un temps et dans un espace, et avec quel langage? Selon quelles stratégies du deuil, quand il s'agit d'un mort, d'un corps, d'un corpus, d'un nom? Encrypter ( «Fors ", dans Abraham, Derrida et Torok 1976). Marquer la présence. Inscrire. Toucher, tenter de retenir, par les chevilles, peut-être, retenir quelque chose, subtiliser un fragment qui deviendra peut-être une relique une fois lancé dans l'économie d'une certaine production symbolique. Le premier geste qui s'offre au deuil est celui d'une mise en scène, d'un décor qui saute aux yeux. C'est la représentation dans le dur, le mort gisant en solide, en pierre, en marbre. $\mathrm{Ou}$ bien Derrida dressé sur un "Catafalque, s.m. Estrade élevée, par honneur, au milieu d'une église, pour recevoir le cercueil ou la représentation d'un mort. [...] Catafalque est le même mot que échafaud (voy. ce mot) " (Glas 1974, 2-3). Balcon, crypte, stèle, piliers, tours, colonne sonore, tour de Babel, phallus tiré par un fil de l'Inde, par la Phrygie, la Syrie, en Grèce, vénéré comme Memnon, «colossale statue résonnante [...] qui produit un Klang sous l'incidence des rayons solaires. Le Klang annonce 
la fin de la religion des fleurs et des colonnes phalliques, mais ce n'est pas encore une voix ou un langage» (Glas 1974, 4a). Théâtre, dramaturgie, architecture sacrée mais rationnelle, genre Panthéon: "aux grands hommes, la Patrie reconnaissante».

La même cérémonie, toujours, mais cette fois dans le privé familier de l'oikos. Le corpus derridien passé par l'épreuve du feu, réduit en cendres, serait recueilli dans une urne funéraire. Serait-elle gardée dans la maison, partie de l'économie et présidant toujours à la hiérarchie, ou serait-elle plutôt glissée dans la niche élégante d'un columbarium? Ou encore, si nous l'osions, les cendres seraient dispersées à tout vent, sur un cours d'eau, sans laisser de traces ni l'espoir d'une reprise, d'un large geste zen ou taoïste. Si nous l'osions encore, nous porterions la main sur le corpus afin de bien le réduire jusqu'à ce qu'il tienne dans nos paumes, au creux même d'une seule main tel ce cercueil de Titus Carmel reproduit ensuite à 127 exemplaires ("Cartouches", dans La vérité en peinture 1978, 212-290). Au moment de nous séparer, nous pourrions nous donner, mutuellement, le corpus à manger, les livres à dévorer, en faire le viatique d'un très long voyage — «Le mot "viatique", je ne l'ai vraiment compris, tu peux traduire “aimé”, qu'au moment où elle m'a dit que tel texte lui serait, tout l'été, un viatique.» (La carte postale 1980, 269), ou enfin, toujours à portée de la main, un vade-mecum, un manuel, c'està-dire, en philosophie, un "poignard de la vérité ».

La première suggestion, la plus courante de l'érection architecturale du philosophe dans son système (Hegel et l'hégélianisme, par exemple, ou même saint Augustin et l'augustinisme; voir Circonfession, dans Bennington et Derrida 1991), Jacques Derrida l'a décrite de tous les cotés dans Glas (1974), nous permettant ainsi d'apprécier les artifices de la construction. La piété des héritiers est libre de s'approcher autrement du mort, de coller autrement à la vérité, sous un autre mode. Du mausolée ou des bandelettes, quelle est la marque de l'affection, de l'intimité, de ce qui touche le plus au propre du mort? Avec quoi entourer son corps, qui n'est plus le corps de Jacques Derrida, mais, disons en vieille langue de clerc, le corpus derridien? Afin d'imprimer un visage non dans la cire ni dans le plâtre, mais dans la douceur d'un tissu. En choisissant d'entourer de bandelettes le corps de Derrida, je me suis placé délibérément en terre de figures, en terrain de tropes. Et c'est donc sous le mode de la poésie, par le biais, l'oblique de la poésie, par sa circularité peut-être de lamentations et de "circonvolutions dansantes" (Circonfession, dans 
Bennington et Derrida 1991, 265) que j'aborde le corps de Jacques Derrida. Aborder ainsi, sans y tomber, l'abîme de cet événement,

faire l'économie de l'abîme: non seulement s'épargner la chute dans le sans-fond en tissant et repliant à l'infini le tissu, art textuel de la reprise, multiplication des pièces à l'intérieur des pièces, mais aussi établir les lois de la réappropriation, formaliser les règles qui contraignent la logique de l'abîme et font la navette entre l'économique et l'anéconomique, la relève et la chute, l'opération abyssale qui ne peut que travailler à la relève et ce qui en elle reproduit régulièrement l'effondrement («Parergon », dans $\mathrm{La}$ vérité en peinture 1978, 44).

Ces parerga, Derrida les a lus chez Kant. En dépit de leur raideur de cadres, d'encadrements ou d'ornements, ces détails esthétiques "traduisent» (il faut retenir ce mot pour en entendre la vibration particulière dans la suite de cet article) des émotions. Ils donnent aux émotions des lieux où se mouvoir et s'émouvoir. "Ce sont en quelque sorte des parerga de la religion dans les limites de la raison pure; elles n'en sont pas des parties intégrantes (sie gehören nicht innerhalb dieselben) mais elles y confinent [aber stossen doch an sie an: elles la touchent, la poussent, la pressent, se pressent contre elle, cherchent le contact, exercent une pression à la frontière]. » ( «Parergon », dans La vérité en peinture 1978, 64-65)

\section{Fichu}

Ces cadres kantiens entourant ces toiles sur lesquelles est peinte la vérité - et en particulier une certaine vérité de la religion encadrée par la raison pure - me mènent à la figure du «fichu », plus souple, plus indiquée pour qui veut caresser un corps aimé. Bout de tissu à peine couvrant, tenant dans la poche ou sur la tête: le fichu n'est certes pas le voile ni le linceul. "Fichu», le mot, on a pu l'entendre porté par un filet de voix, celle d'un mourant, dans l'aveu chagriné qui lui échappe sous un souffle ou un retour de souffle: Atemwende (Paul Celan), si ce n'est «La voix soufflée [sur Antonin Artaud]»(dans L'écriture et la différence 1967): "Je suis fichu». Expression dure à entendre, difficile à dire en parlant de soi ou même en parlant de quelqu'un d'autre, presque vulgaire. Parole quasi inconvenante, incongrue, malséante: "pour "fichu", on dit parfois "foutu” en français, mot qu'on entend aussi bien dans le registre eschatologique de la fin ou de la mort que dans le registre 
scatologique de la violence sexuelle» (Fichus 2002, 36). Jacques Derrida en avait hérité de cette expression: "Je suis fichu!", ce fut la parole ultime de son père. Aveu, constat, confession, soupir. Râle, peut-être. Ce fut aussi l'expression employée sur un certain site Internet pour sonner un glas hostile: «Derrida est fichu! » Je la laisse jouer, cette dissonance, en la reconnaissant comme un fait du contexte de la pensée contemporaine, une certaine liesse autour de la dépouille du "père de la déconstruction ", de celui qui s'exprimait en rébus, c'est-à-dire en «paroles énigmatiques, d'une écriture difficile à lire» ("Rébus » dans Le petit Robert).

Ce fichu, Jacques Derrida l'avait reçu de Walter Benjamin, autre philosophe fichu, fiché, qui avait fiché le camp devant la progression des nazis à sa poursuite. "Walter Benjamin", ainsi que l'écrit Adorno dans le portrait de son maître, c'est «le nom du philosophe qui se suicida en fuyant les sbires d'Hitler» (Adorno 1986a, 201). Qui se suicida dans une géographie de frontières et de montagnes, dans la panique des passages, " esthète fétichiste ou rêveur ",

théoricien politique, militant d'avant-garde, inassimilable d'un côté comme de l'autre, partout rejeté, sans place sur la carte des idéologies européennes, marxiste qu'on accuse de ne pas être le dialecticien qu'il a toujours voulu être, penseur politique auquel on reprochait son messianisme, son mysticisme, son talmudisme. Mal reçu dans son pays et dans son milieu, presque ignoré dans la terre d'exil — la France d'abord et encore aujourd'hui où il a passé sa vie et s'est donné la mort. Homme critique, en position critique, aux limites, homme frontière. ( $+\mathrm{R}$ ", dans La vérité en peinture 1978, 204)

Chez Benjamin, la matérialité de ce bout de tissu avait une importance philosophique apprise de Nietzsche et c'est en tant que tel qu'il l'avait tissé dans la texture de son texte.

L'intuition critique du dernier Nietzsche, selon laquelle la vérité ne s'identifie pas à l'universel atemporel, seule l'histoire étant la figure de l'absolu, fut canonique pour la démarche de Benjamin, qui ne la connaissait peutêtre pas. Le programme est formulé dans une note pour son ouvrage principal, resté fragmentaire: "l'éternel est en tout cas plutôt une ruche attachée à la robe qu'une Idée. » Il ne s'agit pas là d'une simple illustration de concepts par des objets historiques pittoresques [...]. (Adorno, 1986a, 203-204) 
Ainsi qu'Adorno l'ajoute plus loin, «Imperturbablement, il [Benjamin] restait fidèle à son principe selon lequel la plus petite parcelle de réalité perçue vaut le reste du monde»(1986a, 208-209). Ruche d'une robe, fichu, et aussi la singularité d'une main épousée par un gant:

La formule selon laquelle, dans la connaissance, le singulier absolu est l'universel absolu lui va comme un gant. [...] Pour employer une formule, il éprouvait le besoin de rompre avec une logique qui noie le particulier dans l'universel ou n'obtient l'universel qu'en faisant abstraction du particulier. [...] il voulait la [l'essence] deviner de façon méthodique dans la configuration d'éléments éloignés de toute signification. Le rébus devient le modèle de sa philosophie. (1986a, 202)

Tournure de la pensée vers les choses et invention d'un langage, une langue concrète, égyptienne presque ou chinoise: "Le grand enjeu du discours — je dis bien discours — littéraire: la transformation patiente, rusée, quasi animale ou végétale, inlassable, monumentale, dérisoire aussi mais se tournant plutôt en dérision, de son nom propre, rebus, en choses, en nom de choses. La chose, ici, serait la glace dans laquelle prend le chant, la chaleur d'une appellation qui se bande dans le nom.» (Glas $1974,6 b)$ Et puis, «ruche d'une robe », «gant», «fichu », qui «n'est pas n'importe quel mot français pour dire écharpe, châle ou foulard de femme» (Fichus 2002, 11): ce sont des objets proches du corps de la femme dont la féminité ainsi ne serait plus, peut-être, étrangère, inquiétante (unheimlich) pour la pensée, cette «ruche de questions» (Fichus 2002, 38).

\section{Le rêve}

Le fichu qui donne à penser était entré dans l'esprit de Benjamin à l'occasion d'un rêve, c'est-à-dire dans un milieu d'accueil sans frontières, puisque "le rêve est l'élément le plus accueillant au deuil, à la hantise, à la spectralité de tous les esprits et au retour des revenants [...]. Le rêve est aussi un lieu hospitalier à l'exigence de justice comme aux espérances messianiques les plus invincibles.»(Fichus 2002, 36) Mais alors, "Rêvet-on toujours dans son lit, et la nuit? Est-on responsable de ses rêves? Peut-on en répondre?» (11) Ce fichu de rêve, ce fichu rêvé, quelle en était la réalité ? Était-ce un vrai fichu? Faut-il s'éveiller pour accéder au savoir? «Entre rêver et croire qu'on rêve, quelle est la différence? [...] Un rêveur saurait-il d'ailleurs parler de son rêve sans se réveiller? 
Saurait-il nommer le rêve en général ? Saurait-il l'analyser de façon juste et même se servir du mot "rêve" à bon escient sans interrompre et trahir, oui, trahir le sommeil ?» (Fichus 2002, 12) Et Derrida d'imaginer deux réponses à cette question. La première réponse serait celle du philosophe et elle

serait fermement "non" : on ne peut tenir un discours sérieux et responsable sur le rêve, personne ne saurait même raconter un rêve sans s'éveiller. Cette réponse négative, dont on pourrait donner mille exemples de Platon à Husserl, je crois qu'elle définit peut-être l'essence de la philosophie. Ce «non» lie la responsabilité du philosophe à l'impératif rationnel de la veille, du moi souverain, de la conscience vigilante. Qu'est-ce que la philosophie pour le philosophe? L'éveil et le réveil. (Fichus 2002, 12-13)

Il existe cependant une deuxième réponse, "tout autre, mais non moins responsable» : celle

du poète, de l'écrivain ou de l'essayiste, du musicien, du peintre, du scénariste de théâtre ou de cinéma. Voire du psychanalyste. Ils ne diraient pas non, mais oui, peut-être, parfois. Ils acquiesceraient à l'événement, à son exceptionnelle singularité : oui, peut-être peut-on croire et avouer qu'on rêve sans se réveiller; oui, il n'est pas impossible, parfois, de dire, en dormant, les yeux fermés ou grand ouverts, quelque chose comme une vérité du rêve, un sens et une raison du rêve qui mérite de ne pas sombrer dans la nuit du néant. (Fichus 2002, 13)

Et alors, «Y aurait-il une éthique ou une politique du rêve qui ne cède ni à l'imaginaire ni à l'utopie, qui donc ne soit pas démissionnaire, irresponsable et évasive? " (Fichus 2002, 18). La jonction semble impossible de ce que représente le rêve (la musique, la peinture, la littérature, le cinéma) et la philosophie. C'était pourtant le pari de Benjamin qu'Adorno avait repris et que Derrida fait sien en deuxième main:

Adorno écrit ceci, dont je voudrais faire une devise, au moins pour toutes les «dernières fois» de ma vie: «Sous la forme du paradoxe de la possibilité de l'impossible, il [Benjamin] réunit pour la dernière fois la mystique et l'Aufklärung, le rationalisme émancipateur. Il a banni le rêve sans le trahir (ohne ibn zu verraten) et sans se faire le complice de l'unanimité permanente des philosophes, selon laquelle cela ne se peut.» [Adorno 1986a, 21] (Fichus 2002, 19)

Adorno lui-même avait développé plus à fond ce pari de Benjamin. «Le caractère de l'image énigmatique, du rébus, qu'il a lui-même donné 
aux aphorismes de Sens unique et dont tous ses écrits portent la marque, tient à ce paradoxe. La volonté de l'expliciter malgré tout par les seuls moyens dont dispose la philosophie, les concepts, voilà l'un pour l'amour duquel il s'abîmait sans réserve dans le divers. " (Adorno 1986a, 213)

Entre les deux réponses données, celle de la philosophie et celle de l'art (et voire de la psychanalyse), il peut y avoir hésitation, oscillation, aller-retour, "un discours rêveur sur le rêve". Jacques Derrida le reconnaît et aime cette oscillation chez Adorno,

quelqu'un qui n'a cessé d'hésiter entre le «non» du philosophe et le «oui, peut-être, parfois cela arrive » du poète, de l'écrivain ou de l'essayiste, du musicien, du peintre, du scénariste de théâtre ou de cinéma, voire du psychanalyste. En hésitant entre le "non » et le "oui, parfois, peut-être », il a hérité des deux. Il a pris en compte ce que le concept, la dialectique même, ne pouvait concevoir de l'événement singulier, et il a tout fait pour assumer la responsabilité de ce double héritage. (Fichus 2002, 13-14)

"Que nous suggère en effet Adorno ", se demande-t-il ? La conscience éveillée, la philosophie, blesse les plus beaux rêves, elle les macule d'une tache quand "elle nous fait savoir qu'ils sont pure apparence (Schein) au regard de la réalité effective (Wirklichkeit). Les Minima Moralia sont par conséquent sous-titrées: Reflexionen aus dem beschädigten Leben. Non pas "réflexions sur" une vie blessée, lésée, endommagée, mutilée, mais "réflexions depuis ou à partir" d'une telle vie, aus dem beschädigten Leben: réflexions marquées par la douleur, signées par la blessure"(Fichus 2002, 15). Une interrogation de la lumière, venue de la philosophie, se retourne contre elle lorsqu'il s'agit d'" apprendre à vivre enfin» (l'entrevue donnée à Jean Birnbaum [Le Monde] est parue sous ce titre chez Galilée, en 2005). La blessure qui signe et qui saigne impose son tragique à une certaine légèreté de l'être. "Comme toujours chez Adorno, voilà son plus bel héritage, ce fragment théâtral fait comparaître la philosophie en un seul acte, sur une même scène, devant l'instance de tous ses autres. La philosophie doit répondre devant le rêve, devant la musique - représentée par Schubert —, devant la poésie, devant le théâtre et devant la littérature, ici représentée par Kafka.» (Fichus 2002, 16)

Le rêve est donc sans prix. Non comme évasion, fuite, ni même rafraîchissement des capacités de l'esprit. Il vaut à cause de cette vigilance connue de tous les amoureux et de toutes les amoureuses: "moi 
dormant, mon cœur veille» (Cantique des cantiques 5,2, Chouraqui). Cette valeur du rêve est telle qu'elle fait contrepoids à l'appréhension du cauchemar.

Le pire cauchemar (nous pouvons en multiplier les exemples historiques depuis le début du siècle jusqu'à la semaine dernière ${ }^{3}$ ), nous serions donc déçus d'en être réveillés, car il nous aura donné à penser l'irremplaçable, une vérité ou un sens que la conscience risque de nous dissimuler au réveil, voire d'ensommeiller de nouveau. Comme si le rêve était plus vigilant que la veille, l'inconscient plus pensant que la conscience, la littérature ou les arts, plus philosophiques, plus critiques, en tout cas, que la philosophie. (Fichus 2002, 17-18)

La conscience se trompe en nous trompant. Elle se trompe, se fait illusion quant à son éveil, à sa lucidité sinon à sa clairvoyance. Alors que tant de forces s'agitent dans l'accueil du rêve et donc s'amènent à un certain jour, la conscience veut se croire indemne, immune sinon immunisée. Elle risque de nous endormir debout.

Jacques Derrida poursuit sa lecture d'Adorno lisant Benjamin. Il dit avoir trouvé chez eux à la fois ce rêve et cette tâche d'une pensée de la veille et de la vigilance. "Ne pas se laisser impressionner par "l'unanimité permanente des philosophes", à savoir la complicité à rompre et cela même dont il faut commencer par s'inquiéter si l'on veut penser un peu ». Puisque le rêve est accueillant à la fois "à l'exigence de justice comme aux espérances messianiques les plus invincibles» (Fichus 2002, 36), afin de penser, il faut «Bannir le rêve sans le trabir (ohne ihn zu verraten), voilà ce qu'il faut, selon Benjamin $[. .$.$] : se réveiller, cultiver la veille et la$ vigilance, tout en restant attentif au sens, fidèle aux enseignements et à la lucidité d'un rêve, soucieux de ce que le rêve donne à penser, surtout quand il nous donne à penser la possibilité de l'impossible.» (Fichus 2002, 20) Cette injonction paradoxale, Jacques Derrida l'a reçue comme son programme:

La possibilité de l'impossible ne peut être que rêvée, mais la pensée, une tout autre pensée du rapport entre le possible et l'impossible, cette autre pensée après laquelle depuis si longtemps je respire et parfois m'essouffle dans mes cours ou dans mes courses, elle a peut-être plus d'affinité que la

3. Ce discours de Derrida était prononcé le 22 septembre 2001, soit onze jours après le 11 septembre 2001. 
philosophie même avec ce rêve. De cette possibilité de l'impossible, et de ce qu'il faudrait faire pour tenter de la penser autrement, de penser autrement la pensée, dans une inconditionnalité sans souveraineté indivisible, hors de ce qui a dominé notre tradition métaphysique, j'essaie à ma manière de tirer quelques conséquences éthiques, juridiques et politiques qu'il s'agisse du temps, du don, de l'hospitalité, du pardon, de la décision ou de la démocratie à venir. (Fichus 2002, 20-21)

Quelques-uns de ces mots (don, hospitalité, pardon) expriment des réalités que les religions héritières des patriarches connaissent pour en avoir hérité. En sont-elles venues à les traiter de façon routinière? D'autre part, les sociétés civiles en ont fait des droits inscrits dans des chartes. On pourrait croire ainsi que l'impossible a déjà été rendu possible dans les limites des lois et/ou de la raison. Cependant, ce serait, au sens péjoratif cette fois, rêver tout éveillé. La démocratie est encore à venir, les droits inscrits dans les chartes souvent ne sont pas reconnus même dans les cultures héritières des religions abrahamiques. S'ils sont reconnus en quelque lieu, ils ne peuvent recouvrir les exigences rêvées et pourtant irréductibles, impossibles et nécessairement possibles.

\section{Le poème}

Dans le rêve euphorique fait par Benjamin en fuite aux frontières et rapporté par lui, en français, à Gretel Adorno, "Il s'agissait de changer en fichu une poésie " (Fichus 2002, 39). Pour Edmond Jabès, que Derrida a longuement annoté, il s'agit même de changer — et sa suggestion formule à nouveau l'opposition initiale (et la complémentarité) que j'ai posée entre la pierre et le tissu - la pierre en poème. «Entre les morceaux de la Table brisée pousse le poème et s'enracine le droit à la parole. Recommence l'aventure du texte comme mauvaise herbe, hors la Loi, loin de "la patrie des Juifs" qui "est un texte sacré au milieu des commentaires...”. La nécessité du commentaire est, comme la nécessité poétique, la forme même de la parole exilée. Au commencement est l'herméneutique» ("Edmond Jabès et la question du livre », dans L'écriture et la différence 1967, 102). Le poème, surgi du rêve, comme lui illisible mais lisible, veut cependant parler, se faire entendre. Paul Celan s'en émerveille: "Mais un poème, cela parle! Il garde la mémoire de ses dates, mais enfin - il parle. Certes: toujours et seulement en son nom propre, le plus authentiquement propre »(Celan, Le Méridien, trad. par 
J. Launay, cité dans Schibboleth 1986, 118). Le poème acquiert son autonomie absolue, son droit de parole irrévocable. Il l'exerce haut et fort dans le monde, en dépit de l'incompréhension. «Depuis le cœur de sa solitude et à travers son illisibilité immédiate, le poème peut toujours parler — lui-même de lui-même, ici de façon transparente, là selon des tropes ésotériques qui requièrent une initiation et une technique de lecture. Cette auto-référence reste toujours un appel (Anspruch) à l'autre, fût-ce à l'autre inaccessible en soi. Elle ne suspend en rien la référence à l'inappropriable. » (Béliers 2003, 40)

Que faire pourtant, si l'on ne comprend rien ou s'il semble qu'il n'y ait rien à comprendre? Interruptions, suspensions n'empêchent pas la lecture. Pourquoi ? C'est qu'elles sont posées non par le poète ni par le lecteur, mais par le poème lui-même: "Gadamer laisse indécidée, indécidable, sur le seuil, une série de questions qui, loin d'arrêter la lecture interprétative, en ouvrent et libèrent l'expérience même. [...] C'est au poème lui-même, ni au poète ni au lecteur, que se voit alors reconnu le droit de laisser dans l'indécision.» (Béliers 2003, 36) Plus précisément encore

Au-delà d'un trope, Gadamer dit à la lettre que le poème lui-même ne décidera rien. Le poème est bien ici le subjectum que nous évoquions il y a peu. S'il garde une initiative apparemment souveraine, imprévisible, intraduisible, presque illisible, c'est aussi qu'il reste une trace abandonnée, tout à coup indépendante du vouloir-dire intentionnel et conscient du signataire, errant mais de façon secrètement réglée, d'un référent à l'autre et destiné à survivre, dans un "processus infini", aux déchiffrement de tout lecteur à venir. (Béliers 2003, 39)

Le nom de Paul Celan, ses poèmes, sa prose sont également liés à la nuit. "Comme Gadamer j'ai souvent tenté, dans la nuit, de lire Paul Celan et de penser avec lui. Avec lui vers lui» (Béliers 2003, 26). Et ce dont la nuit tient lieu. Ainsi, l'ombre de Celan place son rappel dans celle, impénétrable noirceur, d'Auschwitz. Ses poèmes résistent à cette injonction prononcée par Adorno selon laquelle, après Auschwitz, la poésie aurait été impossible, puisque "même la conscience la plus radicale du désastre risque de dégénérer en bavardage ". Bien plus, "écrire un poème après Auschwitz est barbare, et ce fait affecte même la connaissance qui explique pourquoi il est devenu impossible d'écrire aujourd'hui des poèmes» (Adorno 1986b, 23). Jacques Derrida ne choisit pas Celan 
contre Adorno ni Adorno contre Celan. La résistance à ce dont Auschwitz tient lieu requiert les deux stratégies. Pour ce qui est d'Adorno, Derrida commente: «[...] quoi que signifie ce nom, quels que soient les débats ouverts par les prescriptions d'Adorno à ce sujet [...], dans tous les cas, le mérite indéniable d'Adorno, l'événement unique qu'il aura signé, c'est d'avoir réveillé tant de penseurs, d'écrivains, de professeurs ou d'artistes à leur responsabilité devant tout ce dont Auschwitz doit rester et l'irremplaçable nom propre et la métonymie. » (Fichus 2002, 48)

Quant à Paul Celan, il affirme vigoureusement que le poème parle, qu'il parlera toujours et partout. C'est qu'il parle de sa propre voix, en tant que sujet irréductible. Le poème est l'exacerbation de la langue. La présence de Celan s'incarne dans la chair de ses poèmes, dans cette langue obligatoirement si idiolectale. Le poète n'écrit-il pas «à même la peau de sa langue»(Circonfession, dans Bennington et Derrida 1991, 223)? Son geste poétique, son ductus, c'est-à-dire son trait est un trait incisif tel celui de la circoncision. Le poète scarifie de ses ongles son propre cuir dont il tire un parchemin.

Il arrive aussi que les coups de l'inscription arrivent d'ailleurs. Que l'incision soit donnée, et avec violence. Le poème, posé comme un sceau rougi, porte alors témoignage. Le fichu rêvé portait l'inscription de la lettre «d». Jacques Derrida peut y lire, «[...] si l'on n'exclut pas qu'en ce coup de dés le rêve ait aussi joué [...] le prénom de la première femme de Walter mais encore celui de sa sœur alors très malade: Dora, en grec la peau écorchée, griffée ou travaillée " (Fichus 2002, 42). La peau écorchée, dora, c'est également la peau soumise au fouet, sinon aux verges. Ainsi dans cet épisode tiré des Écritures: "On est allé chercher les apôtres. On les a frappés et on leur a ordonné de ne plus parler du nom de Jésus. Après quoi on les a relâchés. Les apôtres ont quitté le Sanhédrin, joyeux d'avoir été dignes de subir une humiliation pour le Nom.» (Actes des Apôtres 5,40-41) L'écriture dans la chair des martyrs, de ceux et de celles qui se saignent afin de "parler pour ces témoins muets sans parler pour eux, à leur place, et en tirer toutes sortes de conséquences» (Derrida 2000, 13).

\section{La langue}

Le poème trouve son existence dans la langue et la langue ne vit pas sans le poème. "La langue sera d'ailleurs mon sujet: la langue de l'autre, la 
langue de l'hôte, la langue de l'étranger, voire de l'immigrant, de l'émigré ou de l'exilé.» (Fichus 2002, 9) Notre langue maternelle, dans une patrie. Politique:

Son plaidoyer devrait être exemplaire aujourd'hui pour tous ceux qui cherchent, dans le monde, mais en particulier dans l'Europe en construction, à définir une autre éthique ou une autre politique, une autre économie, voire une autre écologie de la langue: comment cultiver la poéticité de l'idiome en général, son chez-soi, son oikos, comment sauver la différence linguistique, qu'elle soit régionale ou nationale, comment résister à la fois à l'hégémonie internationale d'une langue de communication (et pour Adorno, c'était déjà l'anglo-américain), comment s'opposer à l'utilitarisme instrumental d'une langue purement fonctionnelle et communicative sans pour autant céder au nationalisme, à l'État-nationalisme ou au souverainisme État-nationaliste, sans donner ces vieilles armes rouillées à la réactivité identitaire et à toute la vieille idéologie souverainiste, communautariste et différentialiste? (Fichus 2002, 24-25)

D'une part, le privilège de la langue allemande. «Double privilège, quant à la philosophie et quant à ce qui unit la philosophie à la littérature" (Fichus 2002, 26). Alors, comme le dit l'aphorisme célèbre, "les mots étrangers sont les Juifs du langage»(Fichus 2002, 27). Adorno écrit :

Celui qui rentre [sous-entendu: de l'exil] et qui a perdu le contact naif avec ce qui fait sa spécificité [celle de la langue] devra, tout en conservant son intimité avec sa propre langue, faire preuve d'une vigilance infatigable [...] pour échapper à toute supercherie que cette langue pourrait faciliter; il devra éviter de croire que ce que j'aimerais qualifier d'excédent métaphysique de la langue allemande [...] suffit à garantir la vérité de la métaphysique qu'elle propose, ou de la métaphysique en général. [...] Le caractère métaphysique de la langue ne constitue pas un privilège. Ce n'est pas à lui qu'il faut imputer une profondeur qui devient suspecte au moment où elle se glorifie elle-même. [...] Aucun de ceux qui écrivent en allemand et qui savent combien la langue marque leur pensée ne devrait oublier les critiques de Nietzsche à ce sujet. (Adorno 1984, 229, cité dans Fichus 2002, 32-33)

Walter Benjamin et Derrida ont tous les deux cité un texte de Mallarmé qui semble donner une expression quasi parfaite à cette aspiration de la langue à une expression idéale. La difficulté de compréhension épouse, traduit la difficulté du sujet: 
Les langues imparfaites en cela que plusieurs, manque la suprême: penser étant écrire sans accessoire, ni chuchotement mais tacite encore l'immortelle parole, la diversité, sur terre, des idiomes empêche personne de proférer les mots qui, sinon, se trouveraient, par une frappe unique, elle-même matériellement la vérité » (Cité par Benjamin 2000, 255, et dans «Des tours de Babel »1987 , 212).

Une perfection d'expression énigmatique, pourtant. Notre difficulté à lire tient avant tout à notre précipitation vers un contenu, à notre peu de sensibilité au rythme. L'illumination de pensée s'y cache; elle ne se donne pas, elle doit se surprendre aux détours, aux liaisons et aux césures de la syntaxe qui respire. Ce passage de Mallarmé est le condensé d'un débat sur la langue, passerelle vers la traduction ou plutôt la traductibilité, puisque personne "sur terre» ne peut proférer les mots qui, en une seule fois, par la magie d'une frappe unique, se trouveraient être, matériellement, dans le métal de la frappe, la vérité elle-même. Est-ce à dire que la vérité soit alors livrée à des herméneutiques babéliennes, celles de certains dialogues, de certaines discussions, de malentendus englués dans leurs présupposés?

Mais rien ne sert d'invoquer des malentendus quant à l'impression produite par des œuvres de l'esprit. On présuppose par là que la substance d'une pensée existe en soi-même, indépendamment de son destin historique; ou même la connaissance des intentions de l'auteur, par principe inconnaissables, surtout chez un écrivain aussi complexe, aussi réfléchi que Benjamin. C'est à travers les malentendus que se communique l'incommunicable. (Adorno 1986a , 204)

Pas d'illustration ni de défense de la langue qui n'ouvre sur une dimension plus politique. C'est «une mise en garde critique [...]. On y reconnaît un garde-fou indispensable pour l'avenir politique de l'Europe ou de la mondialisation: tout en luttant contre les hégémonies linguistiques et ce qu'elles déterminent, il faudrait commencer par "déconstruire" et les phantasmes onto-théologico-politiques d'une souveraineté indivisible et les métaphysiques états-nationalistes. » (Fichus 2002, 31) C'est-àdire «continuer à aimer la langue allemande, à cultiver cette intimité originaire avec son idiome mais sans nationalisme, sans le "narcissisme collectif" (kollektiven Narzissmus) d'une "métaphysique de la langue". Contre cette métaphysique de la langue nationale, dont on connaît bien la tradition et la tentation, dans ce pays et dans d'autres, la "vigilance", 
dit-il encore, la veille du veilleur doit être “infatigable”.»(Fichus 2002, 31)

D'autre part, intime, secrète mais cependant dans la peau, faiblesse de la langue maternelle apprise dans la terre paternelle. Ses déformations chéries comme chair de la chair.

Si le temps m'en était donné, j’aurais aimé faire plus qu'esquisser une reconstitution; j'aurais exploré une logique de la pensée d'Adorno qui tente de façon quasi systématique de soustraire toutes ces faiblesses, ces vulnérabilités, ces victimes sans défense à la violence, voire à la cruauté de l'interprétation traditionnelle, c'est-à-dire à l'arraisonnement philosophique, métaphysique, idéaliste, dialectique même, et capitalistique. L'exposition de cet être-sans-défense, cette privation de pouvoir, cette vulnérable Ohnmächtigkeit, cela peut être aussi bien le rêve, la langue, l'inconscient, que l'animal, l'enfant, le Juif, l'étranger, la femme. (Fichus 2002, 29-30)

\section{La traduction, le témoignage, la dette}

La langue, qui est toujours plus qu'une langue et plus d'une langue, exacerbée dans le poème, appelle le travail de la traduction. La langue est grosse de la traduction à venir; la traduction la travaille, même dans son monolinguisme.

[...] et notons une des limites des théories de la traduction: elles traitent trop souvent des passages d'une langue à l'autre et ne considèrent pas assez la possibilité pour des langues d'être impliquées à plus de deux dans un texte. Comment traduire un texte écrit en plusieurs langues à la fois? Comment "rendre» l'effet de pluralité ? Et si l'on traduit par plusieurs langues à la fois, appellera-t-on cela traduire? («Des tours de Babel» 1987 , 207-208).

Il ne s'agit pas là d'une question rhétorique, encore moins d'une question technique. Traduire, en effet, c'est survivre. Faire œuvre de survie, entre les frontières, d'une frontière à l'autre. Et cette frontière, l'ultime, la plus lointaine et la plus proche, la plus crainte et la plus subite, la mort.

Survivre au sens courant veut dire continuer à vivre, mais aussi vivre après la mort. À propos de la traduction, Walter Benjamin souligne la distinction entre überleben d'une part, survivre à la mort, comme un livre peut survivre à la mort de l'auteur, ou un enfant à la mort des parents, et, d'autre part, fortleben, living on, continuer à vivre. Tous les concepts qui m'ont 
aidé à travailler, notamment celui de la trace ou du spectral, étaient liés au «survivre» comme dimension structurale. (Entrevue au journal Le Monde, 18 août 2004)

Nous sommes structurellement des survivants, marqués par cette structure de la trace, du testament. Mais, ayant dit cela, je ne voudrais pas laisser cours à l'interprétation selon laquelle la survivance est plutôt du côté de la mort, du passé, que de la vie et de l'avenir. Non, tout le temps, la déconstruction est du côté du oui, de l'affirmation de la vie. (Le Monde, 18 août 2004)

Témoignage, mais radical. La citation de Celan («Aschenglorie») à la fin de Fichus $(2002,57)$ l'exprime fortement:

\section{Niemand \\ zeugt für den \\ Zeugen.}

Dit autrement et sur un autre registre, «il n'y a pas de traduction de la traduction» ( «Des tour de babel» $1987^{\text {b }}, 227$ et 218). Ce n'est pas le retour à un "il faut se taire ", mais au contraire l'ordre (et la promesse) d'une tâche possible de l'impossible. Que l'esthétique mystique d'un nuage d'inconnaissance se traduise en éthique, dette et don.

Ainsi, «la scène de la traduction implique la généalogie ou l'héritage » («Des tours de Babel» 1987 , 227). Héritier et débiteur. «Il [Benjamin] nomme le sujet de la traduction comme sujet endetté, obligé par un devoir, déjà en situation d'héritier, inscrit comme survivant dans une généalogie, comme survivant ou agent de survie. » ( «Des tours de Babel » 1987 , 214) Mais aujourd'hui, non seulement après Auschwitz, après le 11 septembre 2001, après le génocide du Rwanda, après la guerre d'Irak, après le Darfour, après tant de non-événements anonymes, sans noms ni lieux, sans visages, même parfois, hors statistiques. Survivre, témoigner, hériter, être débiteur, responsable. "Qui va hériter, et comment? Y aurat-il même des héritiers? C'est une question qu'on peut se poser aujourd'hui plus que jamais. Elle m'occupe sans cesse.» (Le Monde, 18 août 2004) Il le dit dans la même entrevue. Héritiers non seulement de lui, Jacques Derrida, ni même de ceux de sa "génération »: Lacan, Althusser, Lévinas, Foucault, Barthes, Deleuze, Blanchot, Lyotard, Sarah Kofman, mais aussi la Bible, Platon, Kant, Marx, Freud, Heidegger, etc.

Stupéfiante et archaïque politique de la langue aux frontières qui fait un crime d'un accent, de la légèreté d'un cheveu sur la langue: 
Galaad coupa ensuite à Éphraïm les gués du Jourdain et, lorsqu'un fuyard d'Éphraïm implorait: "Laisse-moi traverser!", ceux de Galaad interrogeaient: «Es-tu éphraïmite, toi ?» et, s’il répondait qu'il ne l'était pas, ordonnaient alors :

Dans ce cas, prononce shibbolèt, pour voir.

Et l'autre, sans réfléchir, spontanément disait:

- Sibbolèt.

On le saisissait alors et on l'égorgeait près des gués du Jourdain. (Juges 12,5-6, Bible Nouvelle traduction)

D'où que vienne le coup qui l'inscrive, une lourde responsabilité se paye dans le sang du poème.

Même là où le poème nomme l'illisibilité, sa propre illisibilité, il déclare aussi l'illisibilité du monde. Un autre poème de Celan commence ainsi: "UNLESBARKEIT dieser / Welt. Alles doppelt. » Et à peine plus loin on hésite à identifier le «tu» que le poème apostrophe: n'importe qui, plus d'un, le poème lui-même, le poète, le lecteur, la profondeur abyssale de telle ou telle autre singularité à jamais encryptée, tout autre, Dieu, toi ou moi («Du, in dein Tiefstes geklemmt...»). (Béliers 2003, 40-41)

Éveil, veille, vigile, vigilance, sentinelle.

\section{Conclusion}

La pudeur et la piété filiale m'ont retenu aux abords de cette nuit où Jacques Derrida n'a pas rêvé qu'il était mort, dont on a pu écrire: «la nuit de vendredi 8 à samedi 9 octobre Jacques Derrida est mort à l'âge de 74 ans». Afin de ne pas l'éveiller ni le trahir, je n'ai rien fait d'autre que de lui rendre la pareille, c'est-à-dire que de découper dans quelques textes qu'il aimait de minces bandelettes qui épousent son corps. Afin de rendre hommage à Jacques Derrida, de reconnaître publiquement ma dette envers lui, j'ai voulu inscrire une sorte de marque personnelle qui fasse signe dans la direction de son œuvre. Je m'en suis tenu à cette étrange, inquiétante matière spirituelle de la langue, plus particulièrement aux langues, à la question de la langue, de la traduction, de la traductibilité, toutes ces questions qui trouvent leur centre dans le poème et qui en surgissent. C'est-à-dire, de la lecture, puisque "Lire, c'est comme traduire », Derrida le rappelle (Béliers 2003, 17; c'est le titre d'un essai de Gadamer: «Lesen ist wie Übersetzen», 1993). 
J'ai repris, de main en main, un bout de tissu, un fichu orné d'images et de la lettre " "d" dont les longueurs effilées décelaient une aspiration extrême vers la spiritualité » (Fichus 2002, 38) surgi d'un rêve de Walter Benjamin. Évocation de cette lettre dans le nom propre: Derrida. Venu du rêve, ce petit carré de tissu a pu contenir des questions capitales pour la théologie, entre autres. Capitales également pour les religions de paroles, faites de liturgie, d'évangélisation, de témoignage, de convictions exprimées, débattues, soutenues, de croyances diverses confiées à des narrations et à des lettres. Langage en actes, surtout, mais non exclusivement par la performativité dans la promesse.

Il serait absurde, comique même, inconvenant, de suggérer qu'un suaire thématique tissé in extremis puisse envelopper le corpus derridien. C'est peut-être à cette tentation d'une thématisation hâtive, pressée, même portée par une piété filiale ou par une (bonne) volonté de vulgarisation que l'injonction du silence imposée par Derrida a voulu couper court. Couper, comme au cinéma, sur une dernière scène. Celle, fameuse, de témoins désarçonnés, des femmes «tremblantes et hors d'elles-mêmes» (Marc 16,8), muettes sur les lieux d'un tombeau vide. Ce que je relève à la fin de mes "circonvolutions dansantes ", ce sont des traits, des entames, sinon des entailles, c'est-à-dire des ouvertures, des cavités mais qui ne sont pas des stigmates ni quelque caverne d'où s'inventerait la fable d'une vérité mythique. Je n'ai rien dit ici du commencement - si ce n'est: «Je m'adresse à vous dans la nuit comme si au commencement était le rêve " (Fichus 2002, 18) - , de l'archie, de la hiérarchie, de l'économie, c'est-à-dire de la maison, du père, de la mère, des filles et des fils. $\mathrm{Ni}$ de la matière, de l'animalité et de l'esprit. Ces plis et replis du corps et de l'esprit, du corps propre et du corps symbolique. De la vérité ellemême, dans ses rapports à la figuration, y compris à la peinture. Et des transports de la figuration (phore) elle-même, y compris sa condensation dans la métaphore, ou ses accompagnements dans la métonymie. Enfin, de l'historicité singulière d'une date qui peut se confier au poème alors que « ce qui a lieu [là], c'est peut-être ce que Celan nomme [...] Geheimnis der Begegnung, le secret de la rencontre» (Shibboleth 1986, 23).

Ces questions que j'égrène, Derrida n'a pas tenté d'y répondre en solitaire. Il les a questionnées, ces questions qui ont l'air de truismes, à partir de et avec les interrogations d'autres philosophes, théologiens, peintres, musiciens, architectes, psychanalystes, écrivains, cinéastes, etc. Il les a souvent expliquées en public, plus ou moins patiemment, avec 
plus ou moins d'humeur, et même sous l'œil impavide de la caméra.

Qu'est-ce que les contemporains (et les théologiens, dans le contexte de cette publication) voudront approfondir en dialogue avec Jacques Derrida, je n'en sais rien. Lui-même, d'ailleurs, en entrevue au monde, hésitait entre deux destins contraires de son opus: dans l'optimisme radieux des Lumières, il entrevoyait une destinée fulgurante; dans la désertification, la mondialisation et la mass-médiatisation de la "pensée ", rien de plus qu'un effet fugace de prêt-à-jeter. Je lui laisse le réitérer:

[...] j'ai simultanément, je vous prie de me croire, le double sentiment que, d'un côté, pour le dire en souriant et immodestement, on n'a pas commencé à me lire, que s'il y a, certes, beaucoup de très bons lecteurs (quelques dizaines au monde, peut-être), au fond, c'est plus tard que tout cela a une chance d'apparaître; mais aussi bien que, d'un autre côté, quinze jours ou un mois après ma mort, il ne restera plus rien. Sauf que ce qui est gardé par le dépôt légal en bibliothèque. Je vous le jure, je crois sincèrement et simultanément à ces deux hypothèses. (Entrevue au journal Le Monde, 18 août 2004)

Traduisez: il n'y a pas de prosélytisme derridien. Reste, à qui veut la recevoir, cette trinité: un don, une dette, une responsabilité.

\section{Références}

Abraham, N., J. Derrida et M. Torok (1976), Cryptonymie: le verbier de l'Homme aux loups, Paris, Aubier Flammarion.

Adorno, T.W. (1984) [allemand 1963], Modèles critiques : interventions, répliques / trad. par. M. Jimenez et E. Kaufholz, Paris, Payot (Critique de la politique).

- (1986a) [allemand 1955], «Portrait de Walter Benjamin", dans Prismes: critique de la culture et société / trad. par. G. Rochlitz et R. Rochlitz, Paris, Payot (Critique de la politique), p. 201-213.

- (1986) [allemand 1955], "Critique de la culture et société », dans Prismes: critique de la culture et société / trad. par G. Rochlitz et R. Rochlitz, Paris, Payot (Critique de la politique), p. 7-23.

Benjamin, W. (2000) [allemand 1972], "La tâche du traducteur ", dans Euvres I / trad. par M. de Gandillac, Paris, Gallimard (Folio Essais 372), p. 244-262. Essai d'abord paru en 1923.

Bennington, G. et J. Derrida (1991), Jacques Derrida, Paris, Seuil. 
Celan, P. (1971) [allemand 1961], Strette: poèmes; suivis du Méridien; et d'Entretien dans la montagne / trad. par J. Daive, Paris, Mercure de France.

Collectif (1981), Les fins de l'homme. À partir du travail de Jacques Derrida / Actes du colloque de Cerisy (juillet-août 1980), sous la direction de P. LacoueLabarthe et J.-L. Nancy, Paris, Galilée.

- (1994), Le passage des frontières. Autour du travail de Jacques Derrida / Actes du colloque de Cerisy (juillet 1992), sous la direction de M.-L. Mallet, Paris, Galilée.

- (1999), L'animal autobiographique. Autour de Jacques Derrida / Actes du colloque de Cerisy (juillet 1997), sous la direction de M.-L. Mallet, Paris, Galilée (Philosophie en effet).

- (2004), La démocratie à venir. Autour de Jacques Derrida / Actes du colloque de Cerisy (juillet 2002), sous la direction de M.-L. Mallet, Paris, Galilée.

Derrida, J. (1967), L'écriture et la différence, Paris, Seuil.

- (1972), Positions. Entretiens avec Henri Ronse, Julia Kristeva, Jean Louis Houdebine, Guy Scarpetta, Paris, Minuit.

- (1974), Glas, Paris, Galilée.

- (1978), La vérité en peinture, Paris, Flammarion.

- (1980), La carte postale de Socrate à Freud et au-delà, Paris - Montréal, Flammarion (Philosophie en effet).

- (1986), Schibboleth pour Paul Celan, Paris, Galilée.

- (1987ª), De l'esprit: Heidegger et la question, Paris, Galilée (Philosophie en effet).

- $\left(1987^{\mathrm{b}}\right)$, «Des tours de Babel », dans Psyché: inventions de l'autre, Paris, Galilée, p. 203-235.

- (2000), Foi et savoir; suivi de Le siècle et le pardon. Entretien avec Michel Wieviorka, Paris, Seuil.

- (2002), Fichus. Discours de Francfort, Paris, Galilée (La philosophie en effet).

- (2002), "Wie recht er hatte! Mein Cicerone Hans-Georg Gadamer», Frankfurter Allgemeine Zeitung (23 mars).

- (2003), Béliers; le dialogue ininterrompu: entre deux infinis, le poème, Paris, Galilée.

— (2005), Apprendre à viure enfin, Paris, Galilée.

Derrida, J., G. Soussana et A. Nouss (2001), Dire l'événement, est-ce possible? / Séminaire de Montréal, pour Jacques Derrida (1 ${ }^{\text {er }}$ avril 1997), Paris Montréal - Budapest - etc., l'Harmattan (Esthétiques).

Gadamer, H.G. (1993), "Lesen ist wie Übersetzen », dans Aesthetik und Poetik, t. I : Kunst als Aussage. Gesammelte Werke Band 8, Tübingen, J.C.B. Mohr (Paul Siebeck), p. 279-285. 
Mallet, M.-L. et G. Michaud, dir. (2004), Jacques Derrida, Paris, L'Herne (Cahiers de l'Herne 83).

Michaud, G. et G. Leroux, dir. (2002), Derrida lecteur, Montréal, Presses universitaires de Montréal (numéro spécial de la revue Études françaises, 38/1-2).

\title{
Résumé
}

Comment parler de Jacques Derrrida? En faisant de cette interrogation un programme. Il s'agira d'en parler à partir de Jacques Derrida lui-même, c'est-à-dire, à partir du corpus derridien (ici: Schibboleth 1986; Fichus 2002; Béliers 2004). Les héritiers doivent découper ce corps avec pudeur, y prélever la peau de bandelettes d'écriture qui se déploient, flottent au vent et caressent. Rien de funèbre, pas de stèle ni de colonne, pas de marbre ni de bronze. Un rêve, un souffle qui demeure dans une chair d'écriture.

\begin{abstract}
How to speak about Jacques Derrida? The question has to be turned into a program. The starting point of a speech about Jacques Derrida has to origin from the derridian corpus itself (here: Schibboleth 1986; Fichus 2002; Béliers 2004). With reverence and restraint, the heirs have to cut this body into pieces and remove carefully the skin of writing strips which unfurl, flutter in the wind and caress. Nothing funereal, no stele nor column, no marble nor bronze. A dream, an everlasting breath in a flesh of writing.
\end{abstract}

